\title{
Dasypoda morawitzi RADCHENKO 2016 (Apoidea - Melittidae - Dasypodaini), une espèce nouvelle pour la faune de France
}

par Guillaume GHISBAIN*, Vladimir G. RADCHENKO** et Denis MICHEZ*

Résumé. Dasypoda morawitzi RADCHEnKo 2016, Melittidae cryptique longtemps confondue avec l'espèce à large distribution Dasypoda hirtipes, est pour la première fois recensée en France.

Abstract. Dasypoda morawitzi RADChEnKo 2016, a cryptic Melittidae mistakenly identified as the large-distribution species Dasypoda hirtipes for a long time, is recorded in France for the first time.

Mots-clés : Dasypoda morawitzi ; Melittidae ; France

$\mathrm{Au}$ sein des Melittidae, l'une des plus petites familles d'apoïdes en termes de nombre d'espèces (MICHENER 2000), se trouve le genre paléarctique Dasypoda LATREILle 1802. Les scopae notablement développées des femelles et la nervation de leurs ailes antérieures, à deux cellules submarginales, distinguent aisément les Dasypoda des autres genres inclus dans la famille des Melittidae. Le recensement le plus récent de la faune d'abeilles de France comptabilise actuellement, pour le genre Dasypoda, 8 espèces (RASMONT et al. 2017b) sur les 28 espèces de la révision ouest-paléarctique de Michez et al. (2004). L'espèce Dasypoda hirtipes (FABRICIUS 1793), largement répandue au sein du Paléarctique attira cependant l'attention de RADCHENKO (2016) qui observa des différences morphologiques notables entre des individus de collection identifiés comme Dasypoda hirtipes. Sur la base de plusieurs caractères morphologiques incluant la pilosité des genitalia des mâles et la morphologie des galéas des deux sexes, RADCHENKo (2016) décrit la nouvelle espèce Dasypoda morawitzi, $29^{\mathrm{ème}}$ espèce de Dasypoda dans la région ouestpaléarctique (RASMONT et al. 2017a) (figure 1).

\footnotetext{
* Laboratoire de Zoologie, Université de Mons, bâtiment

Pentagone, 7000 Mons, Belgique -

guillaume.ghisbain@student.umons.ac.be et denis.michez@umons.ac.be

** Institute for Evolutionary Ecology of the National Academy of Sciences of Ukraine, acad. Lebedev 37, Kyiv 03 I43, Ukraine rvg@nas.gov.ua
}

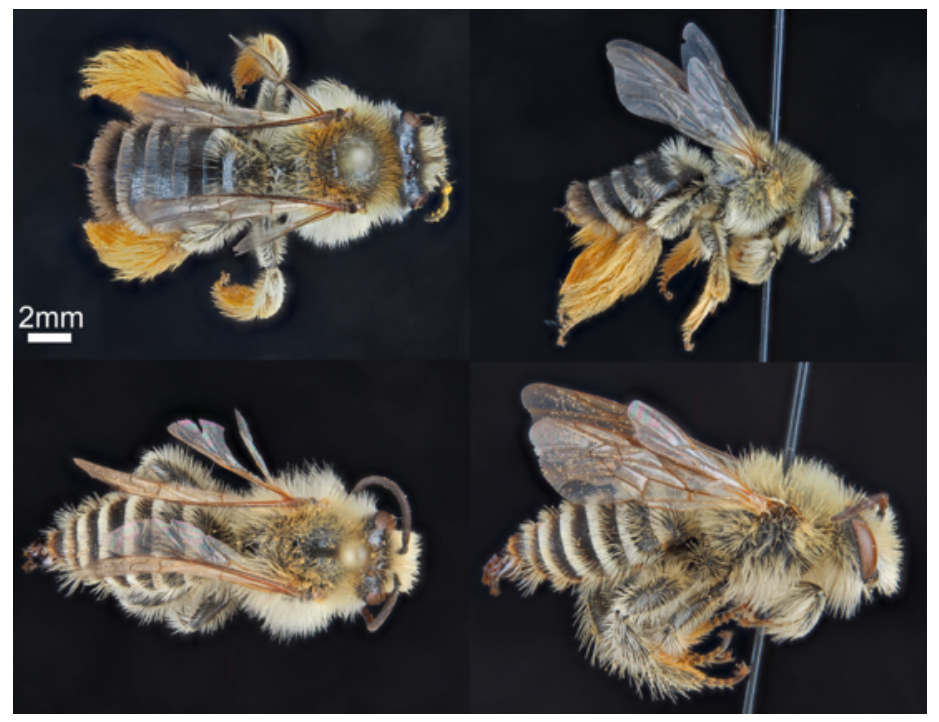

Figure I. Habitus en face dorsale et latérale de Dasypoda morawitzi, femelle en haut, mâle en bas (photos P. Rasmont).

En plus de sa présence en Russie, Ukraine et pays adjacents (RADCHENKO 2016), une publication récente de SCHMID-EGGER et DUBITZKY (2017) recense l'espèce en Europe centrale : l'Allemagne, l'Autriche, ainsi que la Bulgarie sont ajoutées à la distribution de D. morawitzi. L'espèce a aussi été recensée dans les îles grecques orientales (RASMONT et al. 2017a). Nous avons recensé l'espèce pour la première fois en France grâce à des collectes réalisée les 17, 19 et 20 juillet 2017 dans les communes de Peyrins et de Génissieux (département de la Drôme (26)), où mâles et 
femelles furent capturés sur des Asteraceae (habitus illustrés en figure 1). Une étude détaillée des collections du laboratoire de Zoologie de l'Université de Mons nous a également permis d'identifier d'autres spécimens dont les informations relatives à la capture sont détaillées ci-dessous. En France, la distribution de cette espèce semble se limiter à la zone méditerranéenne (figure 2).

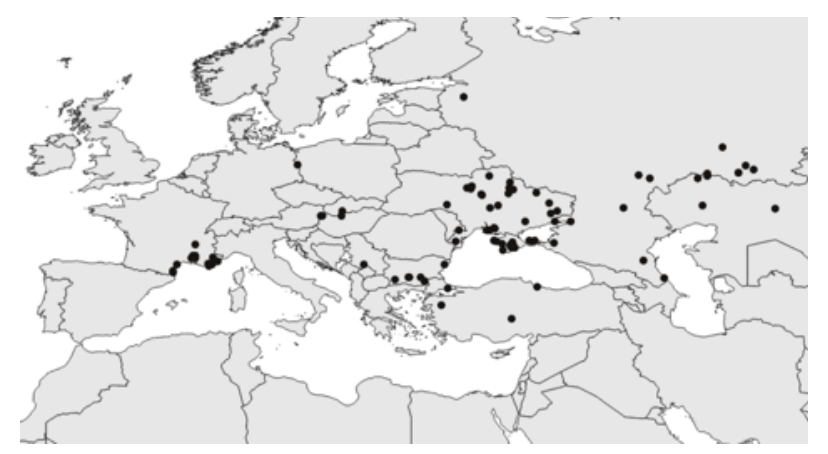

Figure 2. Distribution connue de Dasypoda morawitzi, reprenant les données de Radchenko (2016), Schmid-Egger et Dubitzky (2017) et de la présente étude.

\section{Données additionnelles de distribution et choix floraux}

Sur les 256 spécimens identifiés comme $D$. hirtipes dans les collections de l'Université de Mons, 51 femelles et 27 mâles ont été révisés comme $D$. morawitzi. Les informations de récolte tirées des étiquettes de ces spécimens sont les suivantes : France, Alpes-Maritimes (06), Antibes, mâle récolté le 26 juillet 1962 par J. Mathot ; France, Alpes-de-Haute-Provence (04), mâles récoltés le 18 juillet 1965 entre Annot et Fugeret sur Jasione montana ; France, Alpesde-Haute-Provence, mâle collecté à Annot le 27 juillet 1966 ; France, Var (83), Vidauban (Miquelette), femelles récoltées sur Cichorium intybus le 30 août 1996 par S. Patiny ; France, Var, Plan de la Tour, femelles récoltées sur Crepis vesicaria le 29 août 1996 par S. Patiny ; France, Var, Bormes-les-Mimosas (L'Estagnol), femelle récoltée le $1^{\mathrm{er}}$ juillet 1988 par P. Rasmont sur Cichorieae ; France, Var, Gonfaron, femelle capturée le 23 août 1977 par Rasmont P. et Wéry ; France, Var, Saint-Tropez, femelle récoltée sur C. intybus le 27 août 1996 par S. Patiny ; France, Vaucluse (84), Ile d'Oiselay, femelle récoltée par piège à coupelle bleue le 21 août 2009 par Guilbaud et Paganin ; France, Vaucluse, Courthézon, femelle récoltée par piège à coupelle bleue le 21 août 2009 par Guilbaud et
Paganin ; France, Vaucluse, Lagnes, femelle capturée le 15 juillet 1980 ; France, Vaucluse, Carpentras, mâle collecté sur Malva le 22 juillet 1981 ; France, Gard (30), Aramon, femelle capturée le $1^{\text {er }}$ août 1979 ; France, Hérault (34), Portiragnes-Plage, femelles capturées le 18 juillet 2003 sur Cichorioideae par D. Michez; France, Hérault, Portiragnes-Plage, mâle capturé le 18 juillet 2003 sur Cichorioidae par D. Michez ; France, Pyrénées-Orientales (66), Argelès-plage, femelle capturée le 8 juillet 1965 par J.v.D. Vecht sur Cichorium ; France, Pyrénées-Orientales, Canet, mâle récolté le 3 juillet 1956 ; Serbie, Raska, femelle récoltée le 4 août 1972 par Wahis ; Slovaquie, Levice, femelle capturée le $1^{\text {er }}$ août 1961 par Kocourek ; Slovaquie, Cenkov, mâle capturé le $1^{\text {er }}$ août 1999 par M. Snizek ; Bulgarie, Melnik, mâle capturé le 13 août 1993 par M. Halada ; Bulgarie, Plovdiv, mâles capturés le $1^{\text {er }}$ juillet 1997 par Zaykov ; Bulgarie, Trakia, mâle récolté le 20 juillet 1996 par Zaykov ; Bulgarie, Razlog, mâle capturé le 13 août 1970 par Fraser et Jenkins ; Bulgarie, Trakia, Voïvodine, mâle capturé le 5 août 1997 par Zaykov ; Bulgarie, Svilengrad, mâle capturé le 5 juillet 2000 par M. Snizek ; Bulgarie, Rodopi, Galabovo, mâle capturé le 25 juillet 1997 par Zaykov ; Bulgarie, Zlaté Piesky, mâle récolté en juin 1986 par J.a.M. Halada ; Turquie, Istanbul, Selimpasa, femelles récoltées le 5 juillet 1966 ; Turquie, Samsun, Dikbiyik, mâles capturés le 11 juillet 1967 ; Turquie, Balikesir, Erdek, mâle récolté le 16 juillet 1967 ; Turquie, Istanbul, Selimpasa, mâle collecté le 5 juillet 1966.

Des sessions de collecte de 2017 ont permis de répertorier l'espèce dans deux stations de la Drôme en France : Peyrins, La Savasse (4505’53”N 504'31"E, $275 \mathrm{~m})$, 19.VII.2017 sur Asteraceae (dét. par G. Ghisbain) et Génissieux,

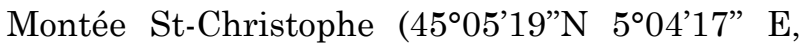
260m), 17.VII.2017 sur Asteraceae (dét. par G. Ghisbain).

Les données issues des collections l'Université de Mons ont révélé la présence de l'espèce en Serbie, en Slovaquie et ont confirmé sa présence en France. Ces informations corroborent également une nette association entre les femelles de cette espèce et la famille des Asteraceae. Les mâles semblent quant à eux interagir avec un panel floral plus important, englobant également des Malvaceae et Campanulaceae. 

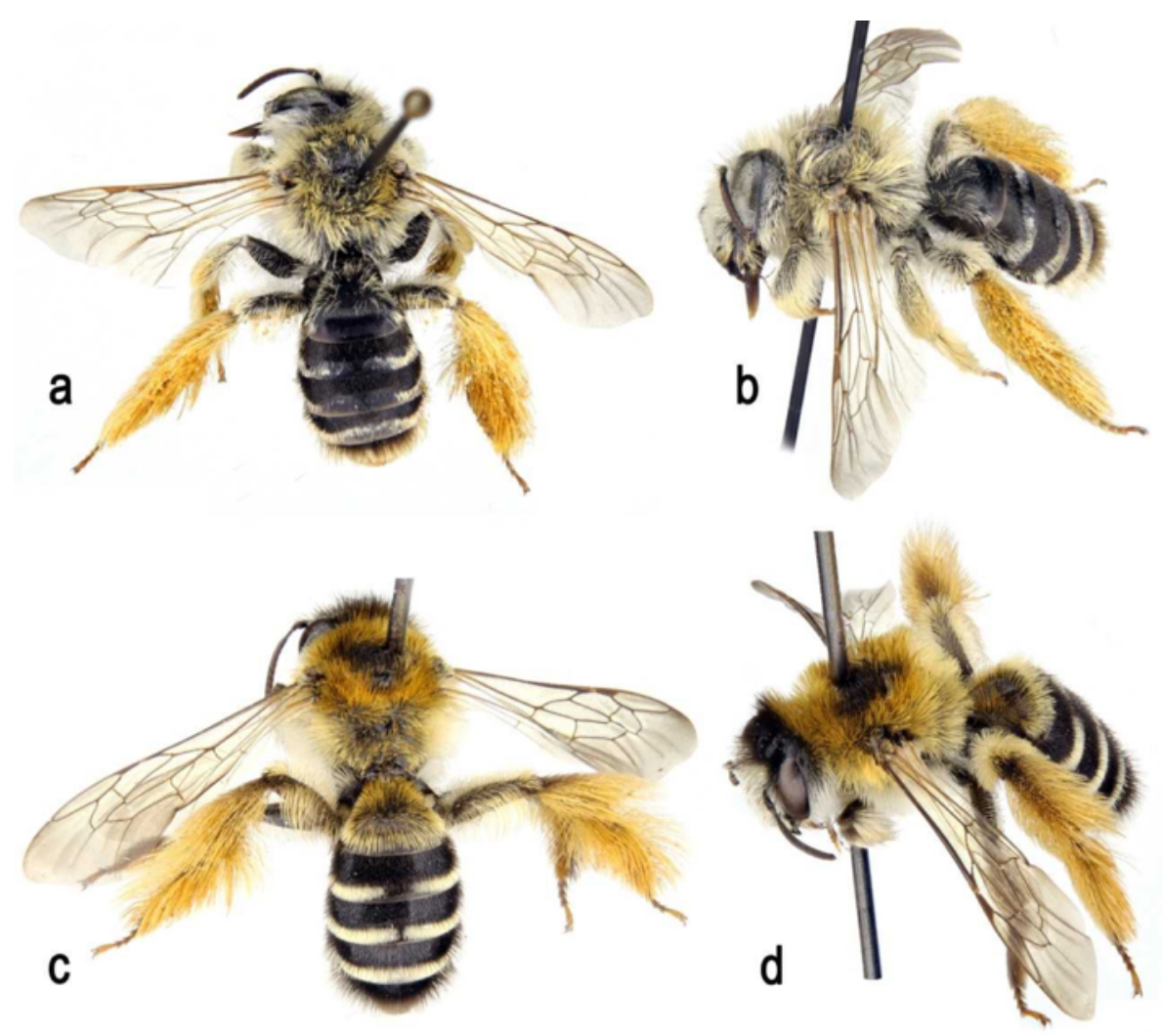

Figure 3. Comparaison des femelles de Dasypoda morawitzi (a, b) et Dasypoda hirtipes (c, d) (photos V. G. Radchenko).

Une révision des spécimens mal identifiés comme $D$. hirtipes dans les collections paléarctiques compléterait la distribution de $D$. morawitzi qui semble vraisemblablement se superposer partiellement à celle de son espèce sœur cryptique. L'identification est relativement aisée sur la base de caractères morphologiques typiques bien décrits par RADCHENKO (2016) (figures 3-4). Notons comme caractère diagnostique net la pilosité des genitalia des mâles, illustrés dans la figure 5 .

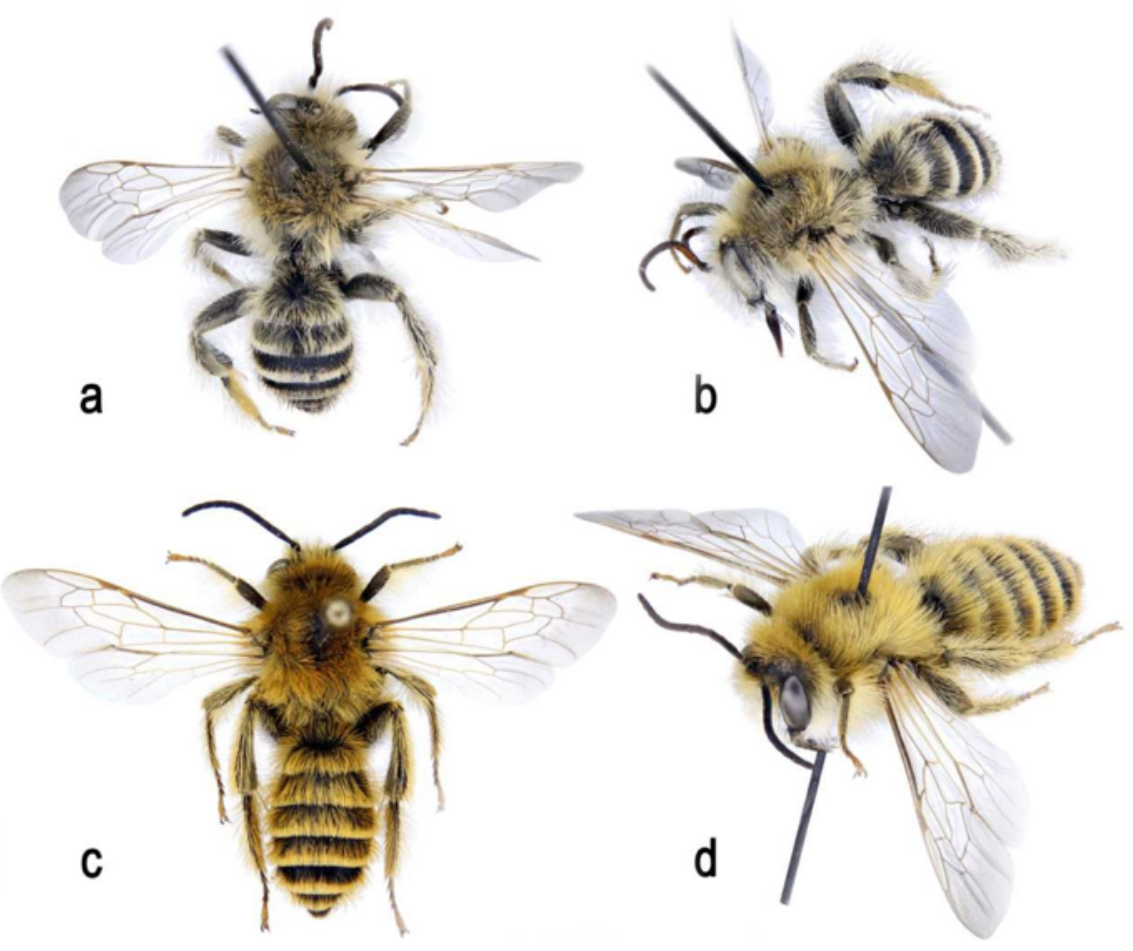

Figure 4. Comparaison des mâles de Dasypoda morawitzi (a, b) et Dasypoda hirtipes (c, d) (photos V. G. Radchenko). 

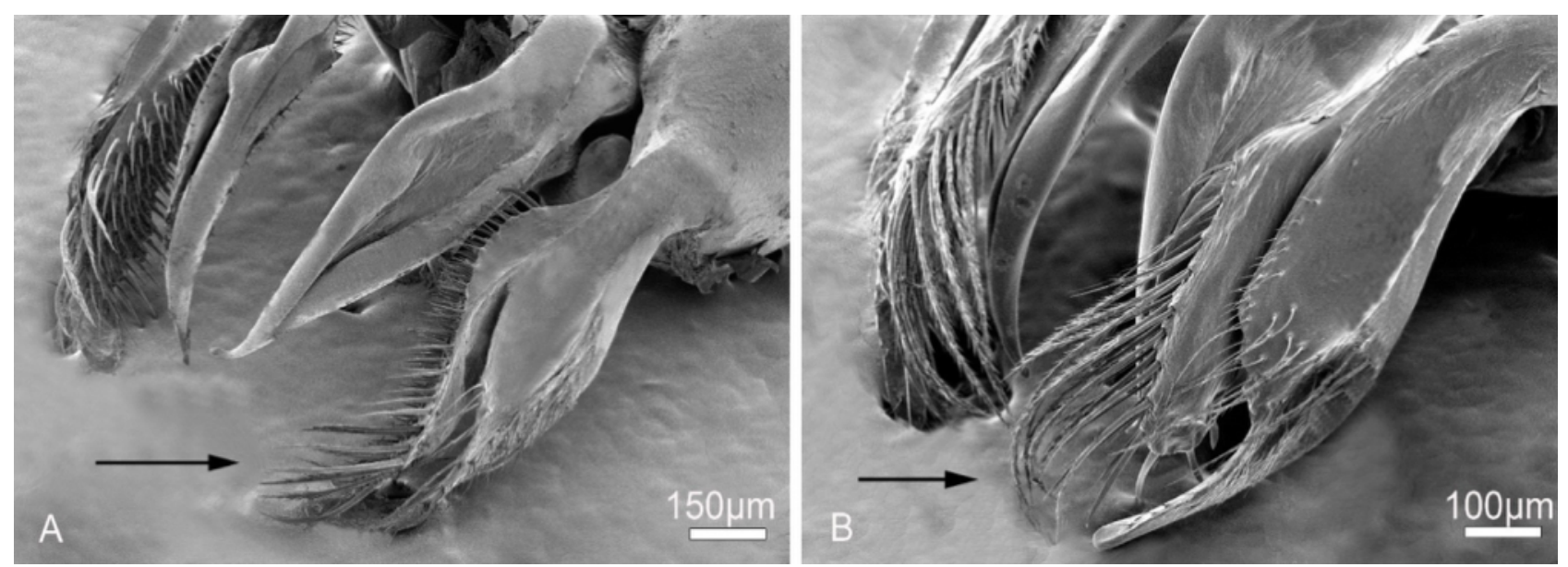

Figure 5. Détail par microscopie électronique des genitalia des mâles de Dasypoda morawitzi (A) et Dasypoda hirtipes (B). Les flèches indiquent la nette différence de pilosité au niveau des gonostyles des deux espèces (photos G. Ghisbain).

\section{Références bibliographiques}

MICHENER CD, 2000. The bees of the world. The Johns Hopkins University Press, Baltimore.

Michez D, Terzo M, RASmont P, 2004. Révision des espèces ouest-paléarctiques du genre Dasypoda Latreille 1802 (Hymenoptera, Apoidea, Melittidae). Linzer Biologische Beitrage 36(2): 847 - 900.

RADCHENKo VG, 2016. A new widespread European bee species of the genus Dasypoda Latreille (Hymenoptera, Apoidea). Zootaxa 4184: 491 - 504.

Rasmont P, Devalez J, Pauly A, Michez D \& RADChenko VG, 2017a. Addition to the checklist of IUCN European wild bees (Hymenoptera: Apoidea). Annales de la Société entomologique de France (N.S.) 53: 17 - 32.

Rasmont P, Genoud D, Gadoum S, Aubert M, Dufrêne E, Le Goff G, MAhÉ G, Michez D \& Pauly A, 2017b. Hymenoptera Apoidea Gallica: liste des abeilles sauvages de Belgique, France, Luxembourg et Suisse. Atlas Hymenoptera, Université de Mons, Mons, Belgium. http://www.atlashymenoptera.net (consulté le 28/02/2018).

SChMid-EgGer C \& Dubitzky A, 2017. Dasypoda morawitzi (Radchenko, 2016) neu für die Fauna von Mitteleuropa (Hymenoptera, Apoidea). Ampulex - Zeitschrift für aculeate Hymenopteren 9: 27 - 31. 